\title{
Indéfinis épistémiques en français et en japonais
}

\author{
Kaneko, Makoto \\ Université d'Okayama (Japon) \\ kaneko06@cc.okayama-u.ac.jp
}

\section{Introduction}

Cette étude ${ }^{1}$ vise à approfondir la connaissance des 'indéfinis épistémiques'. Pour cela, j'examinerai d'une part le quelque en français, quand il est employé au singulier et suivi d'un nom comptable, et d'autre part l'indéfini japonais suivi par un SN casuellement marqué (appelé désormais 'indéfini prénominal'). Les deux formes sont respectivement illustrées par (1a) et (2a) ${ }^{2}$. Les indéfinis épistémiques sont ceux qui servent à exprimer une certaine ignorance sur l'identité du référent. Ainsi, quelque en (1a) indique que le locuteur (ou un autre agent épistémique) ignore de quel chimiste il s'agit, tandis que l'article indéfini un en (1b) permet soit la lecture d'ignorance, soit la lecture d'identification.

(1)a. Quelque chimiste de l'époque avait concocté une concoction pseudo-alimentaire, à base de pétrole peut-être [...] (Muller, 2007 : 145) [*identification / Vignorance]

b. Un chimiste de l'époque avait concocté une concoction pseudo-alimentaire, à base de pétrole peut-être [...] [ Videntification / Vignorance]

(2)a. Dare-ka okyaku-ga zyotyuu-o karakat-teiru. (S. Maruya / cité par Kawaguchi, 1982 : 180)

QUI-KA client-NOM hôtesse-ACC taquiner-PROG ${ }^{3}\left[{ }^{*}\right.$ identification/ Vignorance]

'On ne sait quel client est en train de taquiner une hôtesse d'accueil.'

b. Dare-ka-ga zyotyuu-o karakat-teiru. [ لidentification / لignorance]

QUI-KA-NOM hôtesse-ACC taquiner-PROG

'Quelqu'un est en train de taquiner une hôtesse d'accueil.'

c. Okyaku-ga zyotyuu-o karakat-teiru. [ Videntification / Vignorance]

client-NOM hôtesse-ACC taquiner-PROG

'Un client est en train de taquiner une hôtesse d'accueil.'

Le japonais construit des indéfinis en associant un 'pronom indéterminé' (ex. dare 'qui', nani 'quoi', etc.) avec la particule $k a$ (qui sert soit de marque disjonctive, soit de marque interrogative), comme dare$k a$ en (2a). Lorsque cette combinaison elle-même est casuellement marquée, comme en (2b), elle est neutre en ce qui concerne l'identification du référent, ce qui est aussi le cas pour quelqu'un dans la traduction française. De même, le référent du nom nu, okyaku 'client' en (2c), est soit identifié soit nonidentifié, comme celui d'un chimiste en (1b). D'autre part, en (2a), l'indéfini prénominal, dare-ka, impose la lecture d'ignorance, tout comme le déterminant quelque en (1a) $)^{5}$.

Certaines études récentes ont montré que diverses expressions indéfinies dans d'autres langues (ex. irgendein en allemand, un qualche en italien, algún en espagnol et vreun en roumain) transmettent la lecture d'ignorance, mais que certaines d'entre elles permettent d'autres lectures (lecture de polarité négative et lecture de libre choix) dans des contextes différents. La plupart de ces études ont une approche pragmatique : en réduisant la sémantique de l'indéfini à celle de la disjonction des membres contrastés formant le domaine quantificationnel (que j'appellerai désormais 'alternatives' en empruntant le terme anglais), elles analysent les différentes lectures comme des implicatures conversationnelles dérivées de la 
disjonction d'alternatives. De plus, elles attribuent les distributions divergentes des indéfinis épistémiques aux différentes contraintes imposées au domaine d'alternatives. D'autres études adoptent une approche sémantique, traitant les différentes lectures comme des implicatures conventionnelles. La présente étude soutiendra qu'il faut accepter les deux approches et tenir compte des aspects diachroniques et des propriétés sémantiques de leurs composantes, afin d'expliquer pleinement les propriétés des indéfinis épistémiques du français et du japonais.

Dans ce qui suit, je présenterai d'abord les propriétés distributionnelles communes au quelque du français et à l'indéfini prénominal du japonais. Ces propriétés les distinguent des autres indéfinis épistémiques (Section 2). Je passerai ensuite en revue les approches pragmatique et sémantique des lectures exprimées par les indéfinis épistémiques, et montrerai que la simple application de chacune d'elles aux indéfinis du français et $\mathrm{du}$ japonais suscite des problèmes (Section3). Puis, en profitant des connaissances précédemment accumulées sur les aspects diachroniques de ces deux indéfinis, j'avancerai mes propres hypothèses (Section 4). La section 5 récapitulera les principaux résultats de ce papier.

\section{Propriétés distributionnelles de quelque du français et de l'indéfini prénominal du japonais}

Cette section présentera d'abord une propriété commune aux divers indéfinis épistémiques, et ensuite quelques propriétés qui les différencient.

\subsection{Variation modale}

Une des particularités des indéfinis épistémiques est mise au clair en les comparant avec l'expression $<u n$ $\mathrm{N}$ quelconque $>\mathrm{du}$ français, comme en (3a). Cette expression peut en effet exprimer l'ignorance, comme le montre la faible acceptabilité de $(3 \mathrm{~b})$ où <une partition quelconque $>$ est identifié comme celle de Shostakovich. Il est toutefois à noter que $<u n \mathrm{~N}$ quelconque $>$ interdit non seulement la sélection d'une alternative particulière, comme en (3b), mais aussi l'exclusion d'une alternative, comme le montre la maladresse de $(3 \mathrm{c})$ où une des alternatives, la partition de Shostakovich, est exclue.

(3)a. Le musicien a annoté une partition quelconque. (Jayez \& Tovena, $2010: 55$ )

b. ??Le musicien a annoté une partition quelconque, celle de Shostakovich. (ibid.)

c. ??Le musicien a annoté une partition quelconque, pas celle de Shostakovich. (ibid.)

Pour parler comme Jayez \& Tovena (2010), cette expression est sujette à une contrainte : 'pas de gagnant, pas de perdant'. Cette contrainte revient à dire que toutes les alternatives du domaine (ex. toutes les partitions contextuellement pertinentes en (3a)) peuvent satisfaire la portée de la quantification (ex. «le musisien a annoté $x$ »en (3a)), ce qui est schématisé en (4a) :

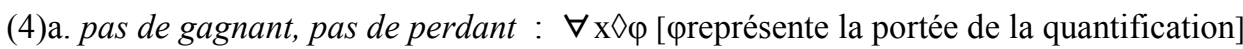

b. pas de gagnant (variation modale) : $\neg \exists \mathrm{x} \square \varphi$

D'autre part, selon Alonso-Ovalle \& Menéndez-Benito (2010), Aloni \& Port (2006) et Fălăuş (2010), quatre indéfinis épistémiques, irgendein en allemand, un qualche en italien, algún en espagnol et vreun en roumain, interdisent qu'une alternative particulière soit sélectionnée (contrainte de pas de gagnant), mais pas nécessairement que toutes les alternatives soient des candidats possibles, c'est-à-dire qu'ils tolèrent l'exclusion d'une alternative particulière. Cette contrainte appelée 'variation modale' est schématisée par (4b). Or, l'acceptabilité de $(5 \mathrm{a}, \mathrm{b})$, où une des alternatives est explicitement exclue, indique que le quelque du français et l'indéfini prénominal du japonais tolèrent tous les deux l'existence d'un perdant, ce qui permet de les ranger du côté des autres indéfinis épistémiques.

(5)a. Yolande a probablement rencontré quelque amie, qui n’était pas Marie. (Jayez \& Tovena, 2008 : 279) 
b. [Dans le contexte où le locuteur prévoit la visite de Taro]

Dare-ka Taro de-wa nai gakusee-ga ki-ta yooda

QUI-KA Taro-COP-TOP NEG étudiant-NOM venir-PST paraître

'Il paraît qu'on ne sait quel étudiant, qui n'est pas Taro, est venu.'

\subsection{Différences distributionnelles de divers indéfinis épistémiques}

Tous les indéfinis épistémiques expriment la lecture d'ignorance et subissent la contrainte de variation modale, mais ils manifestent quelques divergences distributionnelles. Aloni \& Port (2006) récapitulent ces différences en établissant le tableau 1, où la possibilité de quatre lectures est mise à l'épreuve : i) lecture d'ignorance dans une phrase épisodique dénotant un événement spatio-temporellement délimité ; ii) lecture d'ignorance avec la modalité épistémique; iii) lecture de polarité négative avec la négation directe ('clause-mate negation' en anglais) ; iv) lecture non-spécifique de libre choix avec la modalité déontique. Les marques $\sqrt{ }$ et $*$ indiquent respectivement que la lecture en question est admise ou non par chaque indéfini épistémique. Les exemples mentionnés dans le tableau 1 seront cités ultérieurement.

Tableau 1 (adapté d'Aloni \& Port $2006: 4)$

\begin{tabular}{|l|l|l|l|l|}
\hline & épisodique & épistémique & $\begin{array}{l}\text { négation }> \\
\text { (polarité négative) }\end{array}$ & $\begin{array}{l}\text { déontique }>\text { (libre } \\
\text { choix) }\end{array}$ \\
\hline irgendein (allemand) & $\sqrt{ }(10 \mathrm{a})$ & $\sqrt{ }(11 \mathrm{a})$ & $\sqrt{ }(13 \mathrm{a})$ & $(12 \mathrm{a})$ \\
\hline algún (espagnol) & $\sqrt{ }$ & $\sqrt{ }$ & $\sqrt{ }$ & $*$ \\
\hline un qualche (italien) & $\sqrt{ }(17 \mathrm{~b})$ & $\sqrt{ }$ & $*$ & $*$ \\
\hline vreun (roumain) & $*$ & $\sqrt{ }$ & $\sqrt{ }$ & $*(15)$ \\
\hline
\end{tabular}

Le quelque du français et l'indéfini prénominal du japonais réagissent à ces quatre tests de la façon suivante : i) comme on l'a vu en (1a) et (2a), ils autorisent tous les deux la lecture d'ignorance dans une phrase épisodique; ii) les exemples $(6 \mathrm{a}, \mathrm{b})$ ci-dessous indiquent leur compatibilité avec la lecture d'ignorance quand ils figurent avec la modalité épistémique ; iii) on voit en (7a, b) qu'ils sont difficilement compatibles avec la négation directe, et que même dans les cas où ils le sont, ils ne permettent jamais la lecture de polarité négative; iv) il apparaît en $(8 \mathrm{a}, \mathrm{b})$ qu'ils admettent la lecture de libre choix ainsi que la lecture d'ignorance avec la modalité déontique, mais que la lecture de libre choix est facilement supprimée. Il est aussi à noter que le quelque du français préfère nettement la lecture de libre choix à la lecture d'ignorance avec la modalité déontique. Les résultas des quatres tests sont récapitulés dans le tableau 2.

(6)a. Yolande a dû rencontrer quelque amie (\#je sais bien qui c’était). (Jayez \& Tovena, 2008 : 272)

b. Dare-ka otoko-ga yatteki-ta-nitigainai.

QUI-KA homme-NOM venir-PST-devoir épistémique

'On ne sait quel homme a dû venir.' (http://xxxsuzunari88xxx.yamanoha.com/sag_yaku5.htm)

(7)a. *Je n'ai pas encore lu quelque ligne de votre devoir. (Muller, $2007: 144$ )

b. *?Nani-ka oisii mono-o tabe-nai (Yamamori, $2006: 39$ )

QUOI-KA délicieux chose-ACC manger-NEG

'On ne mange pas quelque chose de délicieux.'

(8)a. Il faut avoir quelque protecteur à la cour pour y faire quelque chose qquelque protecteur que ce soit / mais pas n'importe qui \}. (adapté de Van de Velde, $2000: 256$ )

b. Dare-ka otoko-ga goei-no yaku-o hatasa-nakerebaikenai.

QUI-KA homme-NOM garde-GEN service-ACC accomplir-devoir déontique 
\{Dare-demo ii / Dare-demo ii toiu-wake de-wa-nai\}

QUI-même bon / QUI-même bon ce n'est pas le cas

'Un homme ou un autre doit servir de garde. \{N'importe qui est admis. / Ce n'est pas le cas que n’importe qui soit admis.' (adapté de A. Takagi / cité par Kawaguchi, 1982 : 182)

Tableau 2

\begin{tabular}{|l|l|l|l|l|}
\hline & épisodique & épistémique & $\begin{array}{l}\text { négation }> \\
\text { (polarité négative) }\end{array}$ & $\begin{array}{l}\text { déontique }>\text { (libre } \\
\text { choix) }\end{array}$ \\
\hline quelque (français) & $\sqrt{ }(1 \mathrm{a})$ & $\sqrt{ }(7 \mathrm{a})$ & $*(8 \mathrm{a})$ & $\sqrt{(9 \mathrm{a})}$ \\
\hline $\begin{array}{l}\text { indéfini prénominal } \\
\text { (japonais) }\end{array}$ & $\sqrt{ }(2 \mathrm{a})$ & $\sqrt{ }(7 \mathrm{~b})$ & $*(8 \mathrm{~b})$ & $\sqrt{(9 \mathrm{~b})}$ \\
\hline
\end{tabular}

\section{Travaux antérieurs sur les indéfinis épistémiques}

Cette section passera en revue deux approches sur la sémantique des indéfinis épistémiques, pour examiner si elles permettent de rendre compte des distributions du quelque du français et de l'indéfini prénominal du japonais.

\subsection{La lecture d'ignorance définie comme une implicature conversationnelle}

Il est largement admis, dans les études récentes sur les indéfinis, de caractériser un indéfini au moyen des contraintes qu'il impose sur le domain d'alternatives. Kratzer (2005 : 134) suggère effectivement que la fonction essentielle des indéfinis est de changer de domaine ${ }^{6}$.

\subsubsection{Kratzer \& Shimoyama (2002) sur irgendein en allemand}

Dans cette veine, Kratzer \& Shimoyama (2002) examinent la sémantique de irgendein en allemand, en se référant à l'analyse de Kadmon \& Landman (1993) sur any en anglais. Selon Kadmon \& Landman, any exige que le domaine d'alternatives soit maximalement élargi, et son emploi n'est approprié que lorsque l'élargissement du domaine sert au renforcement (i.e. lorsque la proposition au domaine élargi implique celle qui reste sans élargissement du domaine). Ainsi, any en (9a) élargit le domaine des pommes (ex. de l'ensemble des pommes mangeables à l'ensemble plus large incluant les pommes pourries). Dans la phrase négative en (9a), l'élargissement sert à renforcer l'assertion : le fait qu'on n'ait pas de pommes, mangeables ou pourries, implique le fait qu'on n'en ait pas de mangeables. D'autre part, dans la phrase affirmative en (9b), l'élargissement ne conduit pas au renforcement: le fait qu'on ait des pommes mangeables ou pourries n'implique pas le fait qu'on en ait de mangeables, d'où l'inacceptabilité de (9b) :

(9)a. I don’t have any apples. (adapté de Kadmon \& Landman, 1993 : 351)

b. *I have any apples. (ibid.)

Kratzer \& Shimoyama (2002) affirment que irgendein sert, comme any, à élargir le domaine, mais que cet indéfini peut être utilisé non seulement pour renforcer l'assertion, mais aussi pour éviter une assertion fausse. D'après ces auteurs, la lecture d'ignorance, observée dans une phrase épisodique et avec la modalité épistémique, est dérivée pour cette motivation à travers la maxime de quantité, selon laquelle « si Proposition1 (P1) et Proposition2 (P2) sont toutes les deux contextuellement pertinentes et P1 est plus informative que P2, et si le locuteur sait que toutes les deux sont vraies, il doit énoncer P1 plutôt que P2 ». Par exemple, supposons que le domaine élargi de salles en (10) consiste en deux alternatives, la salle de bain et la salle à manger : (10) se réduit à la disjonction de deux propositions alternatives, 'Juan est dans la salle de bain $(\mathrm{A})$ ' et 'Juan est dans la salle à manger $(\mathrm{B})$ '. La proposition A est plus informative que $A \vee B$. Suivant la maxime de quantité, si le locuteur savait à la fois $A$ et $A v B$, il devrait énoncer $A$. Il en est de même pour B et $A v B$. Le fait qu'il énonce $A v B$ plutôt que $A$ ou B nous conduit à inférer qu'il ne sait 
ni A, ni B. En bref, le locuteur évite, au moyen de l'élargissement du domaine, les assertions plus fortes, mais éventuellement fausses :

(10) Juan ist im irgendeinem Zimmer in Haus. (Aloni \& Port, 2006 : 3) [épisodique]

'Juan est dans une salle ou une autre de la maison.'

La même logique donne lieu à l'inférence d'ignorance avec la modalité épistémique, comme en (11) : si irgendeinem Zimmer im Haus dénote, comme en (10), la salle de bain ou bien la salle à manger, (11) se réduit à $\square(A \vee B)$. Or, A et B sont plus informatives que $\square(A \vee B)$. Du fait que le locuteur énonce l'assertion moins informative $\square(A \vee B)$ plutôt que les assertions plus informatives $A$ ou $B$, on peut inférer qu'il ne sait ni A ni B :

(11) Juan muss in irgendeinem Zimmer in Haus sein. (ibid.) [modalité épistémique]

'Juan doit être dans une salle ou une autre de la maison.'

Avec la modalité déontique, comme en (12a), irgendein entraîne non seulement la lecture d'ignorance, mais aussi la lecture de libre choix. Selon Kratzer \& Shimoyama (2002), la dernière lecture est pragmatiquement dérivée pour éviter les fausses inférences d'exhaustivité. Ainsi, si les deux propositions alternatives en (12a), 'vous empruntez livre1' et 'vous empruntez livre2', sont respectivement représentées par $A$ et $B,(12 a)$ se réduit à $\diamond(A \vee B)$, dont les alternatives sont les trois propositions $\diamond(A \wedge B)$, $\diamond \mathrm{A}$ et $\diamond \mathrm{B}$ qui sont toutes plus informatives que $\diamond(A \vee B)$. Par ailleurs, $\diamond \mathrm{B}$ est l'alternative de $\diamond \mathrm{A}$. Du coup, la maxime de quantité stipulant que les informations plus informatives que ce qui est dit sont fausses, apporte, partant de $\diamond \mathrm{A}$, l'inférence d'exhaustivité $\diamond \mathrm{A} \wedge \neg \diamond \mathrm{B}$. Il en est de même pour $\diamond \mathrm{B}$. Par conséquent, la maxime de quantité déclenche, à partir de $(12 a)(=\diamond(A \vee B))$, l'inférence d'exhaustivité (12b), qui contient deux inférences d'anti-exhaustivité (la négation d'une inférence d'exhaustivité), et qui aboutit à l'inférence de libre choix $^{7}$ :

(12)a. Du kannst dir irgendeins von diesen beiden Büchern leihen. (Kratzer \& Shimoyama, $2002: 19)$

'Vous pouvez emprunter un de ces deux livres, mais je ne sais pas lequel.' [ignorance]

'Vous pouvez emprunter un de ces deux livres, n'importe lequel, mais pas tous les deux.' [libre choix]

b. $\quad \operatorname{exhaust}(\diamond \mathrm{A} \vee \mathrm{B})=\diamond(\mathrm{A} \vee \mathrm{B}) \wedge \neg \diamond(\mathrm{A} \wedge \mathrm{B}) \wedge \neg \operatorname{exhaust}(\diamond \mathrm{A}) \wedge \neg \operatorname{exhaust}(\diamond \mathrm{B})$

$$
\begin{aligned}
& =\diamond(\mathrm{A} \vee \mathrm{B}) \wedge \neg \diamond(\mathrm{A} \wedge \mathrm{B}) \wedge \neg(\diamond \mathrm{A} \wedge \neg \diamond \mathrm{B}) \wedge \neg(\diamond \mathrm{B} \wedge \neg \diamond \mathrm{A}) \\
& =\diamond(\mathrm{A} \vee \mathrm{B}) \wedge \neg \diamond(\mathrm{A} \wedge \mathrm{B}) \wedge(\diamond \mathrm{A} \rightarrow \diamond \mathrm{B}) \wedge(\diamond \mathrm{B} \rightarrow \diamond \mathrm{A})=\diamond \mathrm{A} \wedge \diamond \mathrm{B} \wedge \neg \diamond(\mathrm{A} \wedge \mathrm{B}) \text { [libre choix }]
\end{aligned}
$$

Par rapport à la négation directe, irgendein peut avoir une portée étroite, comme en (13a), tout comme la disjonction peut avoir une portée étroite par rapport à la négation, comme en (13b). Dans ce cas, les inférences d'ignorance ou d'anti-exhaustivité, constatées en (10)-(12), ne sont pas observées. Mais cette neutralisation ne cause pas de problème : il est bien connu que, dans un contexte monotone décroissant, comme dans une phrase négative, les implicatures conversationnelles sont annulées.

(13)a. Niemand musste irgendjemand einladen. (idem : 14) [négation]

'Personne ne devait inviter qui que ce soit.' [polarité négative]

b. Personne ne devait inviter Jean ou Marie. = Personne n'a dû inviter ni Jean, ni Marie. 


\subsubsection{Alonso-Ovalle \& Menéndez-Benito (2010) sur algún en espagnol et Fălăuş (2010) sur vreun en roumain}

Alonso-Ovalle \& Menéndez-Benito (2010) et Fălăuş (2010) soutiennent que algún en espagnol et vreun en roumain n'élargissent pas maximalement le domaine, comme any et irgendein, mais minimalement : les deux indéfinis exigent simplement que le domaine ne soit pas singleton (contrainte d'anti-singleton). Ainsi, l'emploi de algún n'est pas approprié lorsque le SN qui le suit est modifié par une expression superlative qui requiert par définition le domaine singleton, comme en (14) :

\#Juan compró algún libro que resultó ser el más caro de la librería.

'Juan a acheté ALGúN livre qui était le plus cher de la librairie.' (Alonso-Ovalle \& Menéndez-Benito, 2010 : 16)

Rappelons-nous ici que les indéfinis épistémiques n'exigent pas que chaque alternative du domaine soit un candidat possible, mais seulement qu'une alternative particulière ne soit pas choisie dans tous les mondes possibles (variation modale). Deux possibilités se présentent ici : i) les variations totale (chaque alternative est un candidat possible) et partielle (au moins une alternative n'est pas un candidat possible) sont toutes les deux admises (pas de gagnant, et éventuellement un perdant) ; ii) la variation totale est exclue (pas de gagnant, et toujours au moins un perdant). Irgendein en allemand, qui permet l'inférence de libre choix, est du premier type. Fălăuş (2010) soutient que le vreun du roumain est du second type et que son incompatibilité avec l'inférence de libre choix dans la modalité déontique, comme en (15), est naturellement réduite au fait que cet indéfini exige lexicalement la variation partielle et non-totale. Par exemple, lorsque le domaine de papiers en (15) contient trois papiers, a, b, c, l'emploi de vreun interdit que les trois membres soient tous des candidats possibles (à savoir, $\neg(\diamond \mathrm{a} \wedge \diamond \mathrm{b} \wedge \diamond \mathrm{c})$ ).

$$
\begin{aligned}
& \text { *Trebuie să trimit vreun articol până mâine diminetă. (Fălăuş, } 2010 \text { : 412) } \\
& \text { Il faut SUBJ écrire.1.SG. VREUN papier avant demain matin } \\
& \text { 'Il faut que j'écrive VREUM papier avant demain matin.' }
\end{aligned}
$$

Algún en espagnol et un qualche en italien seraient du même type et exigeraient la variarion partielle, ce qui est cencé rendre compte de leur incompatibilité avec la lecture de libre choix (voir le tableau 1). Par ailleurs, une telle explication, purement pragmatique, semble laisser mystérieux le fait que un qualche ne permette pas la lecture de polarité négative, à la différence de algún et vreun.

\subsubsection{Quelque en français et indéfini prénominal en japonais}

L'approche pragmatique présentée ci-dessus peut-elle rendre compte des comportements du quelque du français et de l'indéfini prénominal du japonais ? L'analyse de ces deux indéfinis comme une disjonction d'alternatives est effectivement proposée par certains travaux antérieurs. Muller (2007: 143) avance l'idée que «quelque [...] représente une disjonction à partir du singulier ». Dans la même veine sera rangée la remarque de Culioli (1999: 56) selon laquelle «quelque marque un parcours, c'est-à-dire un trajet de point à point, sans que l'on puisse s'arrêter à une valeur stable et assurée ». De même, partant de l'observation que la particule - $k a$ peut servir de marque disjonctive, Okutsu (1996) et Eguchi (1998) paraphrasent l'indéfini prénominal par la disjonction d'alternatives, comme en (16) :

$$
\begin{aligned}
& \text { Jean-ka Paul-ka dare-ka otoko-ga yatteki-ta-nitigainai. } \\
& \text { Jean-soit Paul-soit QUI-KA garçon-NOM venir-PST-devoir }{ }_{\text {épistémique }}
\end{aligned}
$$

'Soit Jean, soit Paul, un garçon ou un autre a dû venir.'

Suivant l'approche pragmatique, la lecture d'ignorance des indéfinis épistémiques du français et du japonais, qui est observée dans une phrase épisodique, comme en (1a) et (2a), ou avec la modalité épistémique, comme en $(6 \mathrm{a}, \mathrm{b})$, est une implicature conversationnelle dérivée de la disjonction. La disponibilité de la lecture de choix libre observée avec la modalité déontique, comme en (8a, b), est alors 
due au fait que ces deux indéfinis permettent que la variation modale soit totale ou partielle, de même que irgendein et à la différence de vreun en roumain. Mais comme je l'ai mentionné en 3.1.2 pour un qualche en italien, l'analyse en termes d'implicature conversationnelle ne semble pas pouvoir rendre compte de l'incompatibilité des indéfinis épistémiques avec la lecture de polarité négative, observée en (7a,b).

\subsection{La lecture d'ignorance définie comme une implicature conventionnelle}

L'autre approche des indéfinis épistémiques attribue leurs distributions divergentes aux différences de leur sémantique lexicale. Ce courant est illustré par Aloni \& Port (2006).

\subsubsection{Aloni \& Port (2006)}

Tout en suivant la tendance récente de l'analyse des indéfinis, selon laquelle leur fonction essentielle est de changer de domaine, Aloni \& Port (2006) postulent que le changement de domaine est effectué non seulement par l'élargissement du domaine, mais aussi par le changement de méthode d'identification : l'identification d'un référent est effectuée, soit par une description soit par un nom propre, soit par un geste démonstratif. Certains indéfinis indiquent que l'identification effectuée n'est pas suffisante dans le contexte. Ainsi, en (17a, b), bien que les exigences de l'identification descriptive (i.e. être le chef du département) soient satisfaites, le contexte exige un autre mode d'identification, de type dénominatif. Irgendein et un qualche mettent en évidence la nécessité de ce changement de méthode d'identification.

$$
\text { description [+identifié] } \rightarrow \text { nom propre [-identifié] }
$$

a. Ich muss hier irgendeinen Professor treffen. Er ist der Direktor vom Institut, aber ich weiss nicht wie er heisst. (Aloni \& Port, $2006: 7$ )

b. Devo incontrare un qualche professor. È il capo del dipartimento, ma non so come si chiama. (idem : 8)

'Je dois voir \{IRGENDEIN / UN QUALCHE \} professeur ici. C'est le chef du département, mais je ne sais pas comment il s'appelle.'

Selon Aloni \& Port (2006), de même que l'emploi d'un indéfini exigeant l'élargissement du domaine n'est approprié que lorsque cet élargissement est motivé (lorsqu'il apporte un renforcement), l'emploi de l'autre type, demandant le changement de méthode d'identification, n'est approprié que lorsque ce changement est motivé (lorsqu'une certaine ignorance est présupposée). D'après cette analyse, la lecture d'ignorance est une implicature «fossilisée qui était pragmatiquement dérivée à l'origine, mais qui est actuellement lexicalisée ${ }^{9} »$, et qui ne peut donc pas être exclue.

Parmi les indéfinis mentionnés ci-dessus, any en anglais ne permet que l'élargissement du domaine, et n'est donc pas accepté dans les phrases épisodiques où l'élargissement n'aboutit pas au renforcement. D'autre part, un qualche en italien n'admet que le changement de méthode d'identification. Il sert donc à exprimer la lecture d'ignorance, dans une phrase épisodique ou avec la modalité épistémique, mais pas celles de polarité négative ou de libre choix, car le changement de méthode d'identification ne sert à rien lorsqu'aucune alternative n'existe, ou lorsque chaque alternative est un candidat possible: comme l'observent Jayez \& Tovena (2008 : 282), il est absurde de signaler l'ignorance à propos d'un objet qui n'existe pas ; comme le note Lefeuvre (2006:162), quand un indéfini exprime lexicalement l'ignorance ${ }^{10}$, « la valeur est forcément spécifique parce que si c'était non-spécifique [comme pour la lecture de libre choix], personne ne saurait de quoi il s'agit et donc il serait absurde de préciser que l'on ne sait pas de quoi il s'agit ».

Cette analyse en termes d'implicature conventionnelle rend bien compte de la distribution de un qualche en italien, qui reste mystérieuse pour l'analyse en termes d'implicature conversationnelle violable. Qu'en est-il pour irgendein ? Selon Aloni \& Port (2006), irgendein permet à la fois l'élargissement du domaine et le changement de méthode d'identification. Cet indéfini sert ainsi soit i) à changer la méthode d'identification pour exprimer la lecture d'ignorance dans une phrase épisodique ou avec la modalité 
épistémique, soit ii) à élargir le domaine pour entraîner la lecture de polarité négative avec la négation directe. Toutefois, l'élargissement du domaine n'aboutit pas toujours à renforcer l'assertion dans la phrase incluant la modalité déontique. Par exemple, en (18), qui inclut la nécessité déontique, si irgendein élargit le domaine de livres de l'ensemble singleton $\{a\}$ à l'ensemble $\{a, b\}$, la lecture de libre choix, à savoir la conjonction de deux permissions, n'implique pas l'obligation :

$$
\begin{aligned}
& \text { Vous devez emprunter IRGENDEIN livre } \rightarrow \text { (conversationally implicate) } \\
& \diamond \text { emprunter a } \wedge \diamond \text { emprunter } \mathrm{b} \wedge \neg \diamond(\text { emprunter a } \wedge \text { emprunter } \mathrm{b}) \text { [cf. (12b)] } \\
& \nrightarrow \text { Vous devez emprunter livre a. ( } \square \text { emprunter a) }
\end{aligned}
$$

Aloni \& Port (2006) suggèrent que ce n'est pas l'élargissement du domaine, mais un effet particulier de l'accent focalisant sur irgendein, qui donne lieu à la lecture de libre choix. Or, algún en espagnol et vreun en roumain permettent tous les deux la lecture de polarité négative, mais pas la lecture de libre choix (voir le tableau 1). L'hypothèse d'Aloni \& Port (2006) nous conduit à supposer que ces deux indéfinis admettent l'élargissement de domaine, de même que irgendein et à la différence de un qualche, mais ne peuvent pas recevoir l'accent focalisant, contrairement à irgendein, pour engendrer la lecture de libre choix.

Aloni \& Port (2006) observent par ailleurs que le changement de méthode d'identification est plus contraint dans les langues romanes que dans les langues germaniques : les indéfinis épistémiques dans les langues romanes obéissent en (19) à la hiérarchie selon laquelle l'identification gestuelle est plus efficace que celle du type dénominatif qui est à son tour plus efficace que celle du type descriptif. Cette hiérarchie n'est pas pertinente pour les langues germaniques.

$$
\begin{aligned}
& \text { Hiérarchie des méthodes d'identification } \\
& \text { geste démonstratif }>\text { nom propre }>\text { description }
\end{aligned}
$$

Ainsi, les exemples (17a, b) ci-dessus et $(20 \mathrm{a}, \mathrm{b})$ ci-dessous montrent que irgendein et un qualche sont tous les deux acceptables i) lorsque l'identification descriptive est satisfaite, mais pas l'identification dénominative, et ii) lorsque l'identification dénominative est satisfaite, mais pas l'identification gestuelle :

$$
\text { nom propre [+identifié] } \rightarrow \text { geste démonstratif [-identifié] }
$$

a. Ich muss hier irgendeinen Professor treffen. Er heisst John Smith, aber ich weiss nicht wie er aussieht. (ibid.)

b. Devo incontrare un qualche professor. Si chiama John Smith, ma non so che aspetto abbia. (ibid.)

'Je dois voir \{IRGENDEIN / UN QUALCHE\} professeur ici. Il s'appelle John Smith, mais je ne sais pas quelle apparence il a.'

D'autre part, (21a, b) vérifient que, lorsque l'identification gestuelle est satisfaite, mais pas l'identification dénominative irgendein est acceptable, tandis que un qualche est inapproprié. Mais la source de ces différences typologiques reste opaque chez Aloni \& Port (2006) :

$$
\text { geste démonstratif [+identifié] } \rightarrow \text { nom propre [-identifié] }
$$

a. Guck mal! Der ist irgendeine Fussballspieler verletzt. Weisst Du wer das ist ? (ibid.)

b. ??Guarda! Un qualche giocatore si è fatto male. Sai che è ? (ibid.)

'Regarde ! \{IRGENDEIN / ??UN QUALCHE \} joueur s'est blessé. Tu sais qui c'est? ‘ 


\subsubsection{Quelque du français et indéfini prénominal du japonais}

Les hypothèses d'Aloni \& Port (2006) ne semblent pas directement applicables au quelque du français et à l'indéfini prénominal du japonais. D'abord, si ces deux indéfinis ne servaient, comme un qualche en italien, qu'à changer la méthode d'identification, ils devraient interdire la lecture de polarité négative et la lecture de libre choix. Mais le quelque du français et l'indéfini prénominal du japonais admettent la lecture de libre choix. Ensuite, s'ils servaient, comme irgendein en allemand, soit à élargir le domaine d'alternatives, soit à changer la méthode d'identification, ils devraient accepter la lecture de polarité négative ainsi que la lecture de libre choix avec l'accent focalisant. Mais les deux indéfinis épistémiques ne tolèrent pas la lecture de polarité négative et permettent la lecture de libre choix sans être accompagnés de l'accent focalisant. En second lieu, l'indéfini prénominal japonais ne manifeste pas la distribution prévue par la hiérarchie des méthodes d'identification en (19): dare-ka prénominal est approprié i) lorsque l'identification descriptive est satisfaite, mais pas l'identification dénominative, comme en (22), tandis que certains locuteurs n'acceptent pas dare-ka prénominal ; ii) lorsque l'identification dénominative est satisfaite, mais pas l'identification gestuelle, comme en (23). Dare-ka prénominal est unanimement accepté ; iii) lorsque l'identification gestuelle est satisfaite, mais pas l'identification dénominative, comme en (24a). D'autre part, le quelque en français n'est pas approprié dans le même contexte, comme en (24b), conformément à la hiérarchie en $(19)^{11}$ :

$$
\begin{aligned}
& \text { description [+identifié] } \rightarrow \text { nom propre [-identifié] } \\
& \text { Marie-wa dare-ka otokonoko-to yakusoku-ga ari-masita. Zibun-no karesi da } \\
& \text { Marie-TOP QUI-KA garçon-avec rendez-vous-NOM avoir-PST soi-GEN copain COP } \\
& \text { to it-teimasi-ta. Demo watasi-ni-wa namae-ga wakari-masen. } \\
& \text { COMP dire-PROG-PST mais moi-DAT-TOP nom-NOM connaître-NEG } \\
& \text { 'Marie avait rendez-vous avec DARE-KA garçon. Elle disait que que c'était son copain, } \\
& \text { mais je ne connais pas son nom.' } \\
& \text { nom propre [+identifié] } \rightarrow \text { geste démonstratif [-identifié] } \\
& \text { \%Marie-wa dare-ka otokonoko-to yakusoku-ga ari-masi-ta. Jean toiu-namae da } \\
& \text { Marie-TOP QUI-KA garçon-avec rendez-vous-NOM avoir-PST Jean s'appeler COP } \\
& \text { to it-teimasi-ta. Demo at-ta koto-ga nai node, dono ko ka } \\
& \text { COMP dire-PROG-PST mais rencontrer-PST fait-NOM NEG parce que quel gars } \mathrm{Q} \\
& \text { wakari-masen. } \\
& \text { savoir-NEG }
\end{aligned}
$$

'Marie avait rendez-vous avec DARE-KA garçon. Elle disait que son nom était Jean, mais comme je ne l'ai jamais vu, je ne peux pas dire de qui il s'agit.'

geste démonstratif [+identifié] $\rightarrow$ nom propre [-identifié]

a. Miro! Dare-ka sensyu-ga kega-o si-teiru. Dare-ka wakaru ? regarde QUI-KA joueur-NOM blessure-ACC faire-résultatif QUI-Q tu sais

'Regarde! DARE-KA joueur s'est blessé. Tu sais qui c'est?'

b. $\quad$ Regarde ! ??Quelque joueur s'est blessé. Tu sais qui c'est?

Cette différence montre que le quelque du français et l'indéfini prénominal du japonais ne manifestent pas toujours la même distribution. 


\section{Hypothèses}

En profitant des études antérieures discutées dans la section 3, et en mettant en avant une perspective diachronique, cette section présentera d'abord mes hypothèses sur l'indéfini prénominal du japonais (4.1), puis quelques remarques spéculatives sur le quelque du français (4.2).

\subsection{Indéfini prénominal du japonais}

Afin d'aborder la sémantique de l'indéfini prénominal, il est à propos de noter que la particule - $k a$ sert en japonais contemporain non seulement de marque disjonctive, comme en (16), mais aussi de marque interrogative : la combinaison à distance d'un pronom indéterminé et de la particule - $k a$ produit une question partielle en (25). Takahashi (1994) fait en outre remarquer que la séquence <pronom indéterminé $+-k a($ ex. dare- $k a)>$ résulte d'une question indirecte, suite à l'ellipse d'un des constituants de la proposition précédente ou suivante (ex. le syntagme verbal correspondant à «taquine l'hôtesse d'accueil » en (26)). Dans ce cas, les marques casuelles nominative (-ga) et accusative (-o), qui sont attachées au pronom indéterminé, peuvent aussi être enlevées :

$$
\begin{aligned}
& \text { Watasi-wa [dare-ga zyotyuu-o karakat-teiru ka] wakara-nai. } \\
& \text { moi-TOP [QUI-NOM hôtesse-ACC taquiner-PROG Q] savoir-NEG } \\
& \text { 'Je ne sais pas qui taquine une hôtesse d'accueil.' }
\end{aligned}
$$$$
\text { Okyaku-ga zyotyuu-o karakat-teiru ga, [dare (-ga)[(...) ] ka] wakara-nai. }
$$$$
\text { client-NOM hôtesse-ACC taquiner-PROG mais QUI-NOM (...) Q savoir-NEG }
$$

'Un client taquine une hôtesse d'accueil, mais je ne sais pas qui.'

La question indirecte incluant un pronom indéterminé et la particule interrogative $k a$ peut être adjointe à la proposition principale de manière parenthétique, comme en $(27 \mathrm{a})$. En se basant sur une étude détaillée des données du japonais ancien et moderne, Kinuhata \& Whitman (2010: 92) affirment que l'emploi indéfini de <pronom indéterminé $+-k a>$ est diachroniquement dérivé d'une question directe parenthétique, incluant l'ellipse et adjointe à la proposition principale. Selon cette analyse, la structure de l'exemple (2a) est représentée par (27b) :

(27)a. Nani-o omot-ta no ka, Taro-wa pinku-no syatu-o katt-ta.

QUOI-ACC penser-PST NF $\mathbf{Q}$ Taro-TOP rose-GEN chemise-ACC acheter-PST

‘À quoi a-t-il pensé ? il a acheté une chemise rose’ (Kinuhata \& Whitman, 2010 : 92)

'Je ne sais pas ce à quoi il a pensé, mais il a acheté une chemise rose.'

b. [question indirecte dare (-ga) [(...)] ka] [proposition principale okyaku-ga zyotyuu-o karakat-teiru]

QUI-NOM Q client-NOM hôtesse-ACC taquiner-PROG

'Je ne sais pas (de) qui (il s'agit), mais un client taquine une hôtesse d'accueil.'

Kinuhata \& Whitman (2010) observent que l'analyse de la séquence <pronom indéterminé $+-k a>$ en termes de question indirecte parenthétique n'est possible que lorsque la proposition à laquelle elle est adjointe est une assertion, alors que l'analyse comme indéfini (équivalent à la disjonction d'alternatives) est clairement identifiée quand cette séquence figure dans une phrase non-assertive (ex. question, ordre, demande, proposition, etc.), comme en $(28 \mathrm{a}, \mathrm{b})$ :

(28)a. Nani-ka motte-mairi-masyoo. [indéfini argumental]

QUOI-KA emporter-venir-je vous propose

'Je vous propose d'emporter quelque chose.' (Keiseikaityuukagami publié en 1818 / cité par Kinuhata \& Whitman, $2010: 95)$ 


\author{
Dare-ka gakusee san-o okurikon-dekure-mase-n-ka $?^{12}$ [indéfini prénominal] \\ QUI-KA étudiant Mr-ACC envoyer-donner-COP-NEG-Q
}

'Pourriez-vous nous envoyer un étudiant ou un autre? (quelque étudiant que ce soit)'

Kinuhata \& Iwata (2010) montrent de plus que les premières occurrences du $<$ pronom indéterminé $+-k a>$, qui est indéniablement du type non-interrogatif, ne datent que du début du $19^{\text {ème }}$ siècle. En profitant de ces connaissances accumulées, j'avancerai l'hypothèse (29) selon laquelle la suite <pronom indéterminé $+\mathrm{KA}>$ peut recevoir deux interprétations en japonais contemporain.

Hypothèse

La suite <pronom indéterminé $+-k a>$ dans une position prénominale peut être interprétée ;

(i) soit comme incluant la particule interrogative $-k a$ et comme équivalant sémantiquement à la question indirecte parenthétique, lorsque cette interprétation est possible ;

(ii) soit comme incluant la particule disjonctive $-k a$ et comme équivalant sémantiquement à la disjonction d'alternatives, lorsque l'interprétation (i) n'est pas disponible.

Suivant (29), dans une phrase assertive épisodique ou incluant la modalité épistémique, l'interprétation (i) est posssible, et la lecture d'ignorance est obtenue en tant qu'implicature conventionnelle ou présupposition pour énoncer une question, car le locuteur ne pose une question sur l'identité d'une entité que lorsqu'il l'ignore. Ensuite, dans une phrase négative, comme en (30a), l'interprétation (i) entraîne la lecture d'ignorance, mais non la lecture de polarité négative : il n'est pas concevable qu'une question soit posée sur l'identité d'une entité dont l'existence est explicitement niée. L'interprétation (ii) n'entraîne pas non plus la lecture de polarité négative, car la disjonction exprimée par $-k a$ en japonais n'est jamais utilisée pour exprimer la négation totale, en raison de la concurrence d'une autre particule, mo, qui se spécialise pour la quantification universelle, comme le montre (30b). Corrélativement, la lecture équivalente à celle de polarité négative est assumée par dare-mo, plutôt que dare-ka, comme en (30a) :

(30)a.

$$
\begin{aligned}
& \text { Dare-ka gakusee-ga ko-nakat-ta / Dare-mo gakusee-wa ko-nakat-ta } \\
& \text { QUI-KA étudiant-NOM venir-NEG-PST / QUI-MO étudiant-TOP venir-NEG-PST }
\end{aligned}
$$

'Je ne sais quel étudiant n'est pas venu. / Quant aux étudiants, ils ne sont pas venus.'

b. Marie-ka Jean-ka-ga ko-nakat-ta. / Marie-mo Jean-mo ko-nakat-ta.

Marie-KA Jean-KA-NOM venir-NEG-PST / Marie-MO Jean-MO venir-NEG-PST

'Soit Marie soit Jean n’est pas venu.' / Quant à Marie et Jean, ils ne sont pas venus.'

Enfin, avec la modalité déontique, comme en (31), la lecture d'ignorance est obtenue suivant l'interprétation (i), tandis que la lecture de libre choix, qui rend la question sur l'identité du référent peu significative, doit être pragmatiquement dérivée suite à l'interprétation (ii) : l'indéfini prénominal du japonais n'impose pas, comme irgendein en allemand et à la différence de vreun en roumain, la contrainte d'une variation modale obligatoirement partielle. N'étant dérivée que pragmatiquement, cette lecture peut être exclue, comme le montre la possibilité de la suite correspondant à "Mais pas n'importe qui. » en (31b). Il en est de même pour la phrase non-assertive exprimant une demande en (28b).

$$
\begin{aligned}
& \text { Dare-ka otoko-ga goei-no yaku-o hatasa-nakerebaikenai }(=(8 b)) \\
& \text { QUI-KA homme-NOM garde-GEN service-ACC accomplir-devoir }{ }_{\text {déontique }}
\end{aligned}
$$

a. 'Je ne sais quel homme doit servir de garde.' [interprétation (i)]

b. 'Un homme ou un autre doit servir de garde \{N'importe qui est bien / Mais pas 


\section{n’importe qui\}.’ [interprétation (ii)]}

Or, la lecture d'ignorance pourrait aussi être dérivée pragmatiquement, suite à l'interprétation (ii), tandis que (29) stipule que l'interprétation (i) et non pas celle de (ii), est responsable de cette lecture. Deux arguments viennent étayer cette hypothèse. D'abord, comme nous l'avons vu dans la section 3.2.2, le dare-ka prénominal impose une restriction aux méthodes d'identification permises : il est approprié i) lorsque le locuteur identifie la personne en question par une information descriptive, mais non par son nom propre (ex. (22)), et ii) lorsque le locuteur peut la désigner, mais ne connaît pas son nom (ex. (24a)), tandis qu'il est inapproprié, au moins pour certains locuteurs natifs ${ }^{13}$, iii) lorsque le locuteur connaît son nom, mais n'arrive pas à la désigner (ex. (23)). Or, comme les exemples (32)-(34) l'indiquent, les cas où le dare-ka prénominal est approprié ou inapproprié correspondent exactement aux cas où la question incluant le pronom indéterminé dare est appropriée ou inappropriée : en japonais, l'emploi interrogatif de dare 'qui' sert essentiellement à demander le nom d'une personne :

$$
\begin{aligned}
& \text { description [+identifié] } \rightarrow \text { nom propre [-identifié] } \\
& \text { Marie-wa otokonoko-to yakusoku-ga ari-masita. Zibun-no karesi da to } \\
& \text { Marie-TOP garçon-avec rendez-vous-NOM avoir-PST soi-GEN copain COP COMP } \\
& \text { it-teimasi-ta. Demo watasi-ni-wa dare-ka wakari-masen. } \\
& \text { dire-PROG-PST mais moi-DAT-TOP QUI-Q savoir-NEG }
\end{aligned}
$$$$
\text { 'Marie avait rendez-vous avec un garçon. Elle disait que que c'était son copain, mais je }
$$
ne sais pas de qui il s'agit.'

$$
\begin{aligned}
& \text { nom propre [+identifié] } \rightarrow \text { geste démonstratif [-identifié] } \\
& \text { \%Marie-wa otokonoko-to yakusoku-ga ari-masita. Jean toiu-namae da to } \\
& \text { Marie-TOP garçon-avec rendez-vous-NOM avoir-PST Jean s'appeler COP COMP } \\
& \text { it-teimasi-ta. Demo at-ta koto-ga nai node, dare ka wakari-masen. } \\
& \text { dire-PRPG-PST mais rencontrer-PST fait-NOM NEG parce que QUI Q savoir-NEG }
\end{aligned}
$$

'Marie avait rendez-vous avec un garçon. Elle disait que son nom était Jean, mais comme je ne l'ai jamais rencontré, je ne peux pas dire de qui il s'agit.'

$$
\begin{aligned}
& \text { geste démonstratif [+identifié] } \rightarrow \text { nom propre [-identifié] } \\
& \text { Miro! Sensyu-ga kega-o si-teiru. Dare-ka wakaru ? } \\
& \text { regarde joueur-NOM blessure-ACC faire-résultatif QUI-Q tu sais }
\end{aligned}
$$

'Regarde ! Un joueur s'est blessé. Tu sais qui c'est?'

En second lieu, Kawaguchi (1982) fait remarquer que le dare-ka prénominal est suivi par le SN koziki 'clochard' en (35a) plus difficilement que par le SN zyoyuu 'actrice' en (35b). Afin de rendre compte de cette différence, Kawaguchi (1982: 176) affirme que «le SN suivant le dare-ka doit dénoter des membres facilement individualisés en vue des connaissances encyclopédiques ». L'origine de cette restriction lexicale n'est toutefois pas claire :

\footnotetext{
(35)a. ?Taro-wa dare-ka koziki-o mikake-ta. (Kawaguchi, $1982: 176$ )

Taro-TOP QUI-KA clochard-ACC voir-PST

'Taro a vu je ne sais quel clochard.'

b. Taro-wa dare-ka zyoyuu-o mikake-ta. (ibid.)

Taro-TOP QUI-KA actrice-ACC voir-PST
} 
'Taro a vu je ne sais quelle actrice.'

Or, le même contraste d'acceptabilité entre le clochard et l'actrice est observé dans les questions partielles au moyen de dare, comme en $(36 \mathrm{a}, \mathrm{b})$ : il est moins concevable de demander l'identité d'un clochard que celle d'une actrice, ce qui appuie l'hypothèse de (29) selon laquelle la lecture d'ignorance est due à l'interprétation (i) du dare-ka prénominal comme une question :

(36)a.
Tharo-ga mikake-ta koziki-wa dare ka ?
'Qui est le clochard que Taro a vu ?'
b. $\quad$ Taro-ga mikake-ta zyoyuu-wa dare ka ?
Taro-NOM voir-PST actrice-TOP QUI-Q
'Qui est l'actrice que Taro a vue?'

L'hypothèse que l'interprétation (ii) n'est pas responsable de la lecture d'ignorance en (35b) est vérifiée par le fait que le contraste d'acceptabilité entre le clochard et l'actrice n'est pas observé, comme en (37a, b), avec la lecture de libre choix, dont l'interprétation (ii) est sûrement responsable :

(37)a.

$$
\begin{aligned}
& \text { Dare-ka koziki-o mituke-tekudasai.Tyoosa-ni hituyoo-desu. } \\
& \text { QUI-KA clochard-ACC trouver-donner investigation-pour nécessaire-COP }
\end{aligned}
$$

'Trouvez-moi un clochard ou un autre ! C'est nécessaire pour mon enquête.'

'Trouvez-moi une actrice ou une autre ! C'est nécessaire pour mon enquête.'

\subsection{Remarques spéculatives sur le quelque du français}

Une idée analogue, celle de la coexistence de différentes interprétations dans des environnements différents, semble envisageable pour quelque en français. En effet, selon les travaux antérieurs, au moins trois facteurs contribuent à la constitution de la sémantique de quelque. D'abord, à propos d'une série d'expressions incluant quelque (non seulement quelque au singulier, mais aussi quelques au pluriel, quelqu'un, quelque chose, etc.), Corblin (2010b : 14) soutient que leur vocation essentielle commune est d'affirmer, s'il n'y a pas de quantifieur explicite, la quantification existentielle. Avec un quantifieur qui ne contredit pas cette vocation existentielle, comme en général en (38), quelque peut avoir une portée étroite, donnant lieu à la lecture de libre choix. La lecture de polarité négative sous la portée de la négation directe, qui se trouve à l'antipode de la vocation existentielle, est donc exclue. Corblin (2010b : 13) suggère aussi que la vocation existentielle de la série d'expressions incluant quelque est « indépendante de la charge épistémique du déterminant singulier quelque ».

Quelque chose a en général un prix. (Corblin, 2010b : 4) [ $\sqrt{ }$ lecture de libre choix]

En second lieu, concernant la charge épistémique, Jayez \& Tovena (2008) soutiennent qu'elle n'est qu'un effet dérivé, et que la sémantique lexicale du quelque singulier consiste à marquer que le locuteur invoque, pour asserter la proposition, une inférence plutôt qu'une évidence perceptuelle ou une information transmise par les autres. Par exemple, en (39a), bien que l'identité de l'amie en question soit inconnue, l'emploi de quelque n'est pas approprié, car la proposition "Yolande a rencontré une amie » n'est fondée que sur l'information transmise par elle-même, et aucune inférence n'intervient. En revanche, (39b) est accepté, la proposition «un idiot a oublié d'éteindre » étant inférée suite à la perception de la lumière.

(39)a. ??Yolande m'a dit qu'elle avait rencontré quelque amie. (Jayez \& Tovena, $2008: 272$ ) 
b. Il y a de la lumière dans le bureau : quelque idiot a oublié d'éteindre. (idem : 274)

Cette analyse de quelque comme une marque d'évidentialité inférentielle explique en outre pourquoi cet indéfini n'est pas acceptable en (24b), où le locuteur indique le référent par un geste démonstratif, mais n'est pas au courant de son nom : le locuteur n'a besoin de recourir à aucune inférence pour décrire un événement qui se déroule sous ses yeux. Selon Jayez \& Tovena (2008 : 275-276), dans une situation conforme à la contrainte d'inférence, la lecture d'ignorance est le plus souvent naturelle. Toutefois, n'ayant pas de relation logique avec cette contrainte, elle n'est observée que par défaut.

Troisièmement, un facteur diachronique semble aussi pertinent pour la sémantique de quelque. Comme Muller (2007 : 139-140) le fait remarquer, le déterminant quelque est issu de la combinaison de l'adjectif interrogatif quel et du complémenteur que, comme en (40) : "quel était en contact avec la subordonnée de concession ", et "l'emploi de quelque dans les concessives est toujours courant» en français contemporain, comme dans l'expression, «quelque étudiant que ce soit... ».

$$
\begin{aligned}
& \text { quel samblant qu'el en feîst (Foulet, } 1982: 190 \text { ) } \\
& \text { 'quel semblant qu'elle en fasse' }
\end{aligned}
$$

Discutant sur la constuction du type «quelque étudiant que ce soit... », Corblin (2010a : 31-32) soutient que «l'origine de l'universalité forte [correspondant à la lecture de libre choix] est dans la complétive au subjonctif disjonctive ». Dépourvu de complétive au subjonctif, le quelque au singulier n'entraîne certes pas lui-même la lecture de libre choix, qui doit donc être pragmatiquement dérivée de la disjonction d'alternatives, et qui ne contredit d'ailleurs ni l'évidentiatité inférentielle, ni la vocation existentielle. Toutefois, le fait que cette lecture soit préférée à la lecture d'ignorance avec la modalité déontique (voir l'exemple (8a)) sera attribué à la persistance de la sémantique lexicale de la construction d'origine, en raison « du caractère plutôt écrit et soutenu du quelque au singulier» (Muller, 2007 : 135).

En bref, les propriétés distributionnelles de quelque, un peu mystérieuses par rapport aux indéfinis épistémiques dans les autres langues européennes, semblent provenir des facteurs hétérogènes qui constituent sa sémantique : i) sa sémantique lexicale d'évidentialité inférentielle entraîne, par défaut, la lecture d'ignorance ; ii) la vocation existentielle commune à la série d'expressions incluant quelque exclut la lecture de polarité négative ; iii) la lecture de libre choix n'est qu'un effet pragmatiquement dérivé, mais est préférée peut-être en raison de la persistance de la sémantique lexicale de sa source diachronique, quelque $N$ que ce soit.

\section{Remarques récapitulatives}

Après avoir passé en revue les approches pragmatique et sémantique des indéfinis épistémiques, cette étude a soutenu qu'aucune de ces deux approches n'explique pleinement à elle seule les comportements des deux indéfinis épistémiques du français et du japonais, et par ailleurs que leurs distributions apparemment identiques sont des résultats dus à des facteurs différents.

Pour l'indéfini prénominal du japonais qui consiste en $<$ pronom indéterminé $+-k a>$, il a été soutenu que : (i) étant donné l'ambiguïté de la particule $-k a$ (disjonction et question), cette suite est interprétée soit comme une question indirecte parenthétique, soit comme une disjonction d'alternatives; (ii) la lecture d'ignorance dans une phrase épisodique, ou avec la modalité épistémique, est une implicature conventionnelle associée à l'interprétation interrogative de cette suite, qui est préférée à l'autre interprétation pour une raison diachronique ${ }^{14}$; (iii) la lecture de polarité négative n'est générée ni par l'interprétation interrogative, ni par l'interprétation disjonctive, étant donné a) l'incongruité de demander l'identité d'un référent dont l'existence est explicitement niée et b) la portée nécessairement large de -ka disjonctif par rapport à la négation directe ; (iv) la lecture de libre choix avec la modalité déontique est une implicature conversationnelle dérivée pragmatiquement suite à l'interprétation disjonctive.

Pour le quelque au singlulier du français, il a été suggéré, en profitant des analyses antérieures, que : i) tout en étant partiellement lexicalisée, sa lecture d'ignorance n'est qu'un effet de sa relation étroite avec l'évidentialité inférentielle qui constitue le noyau de sa sémantique lexicale ; ii) l'impossibilité de la 
lecture de polarité négative doit être attribuée à son incompatibilité avec la vocation existentielle commune à la série d'expressions incluant quelque, y compris le quelques au pluriel, quelqu'un et quelque chose ; iii) la lecture de libre choix est une implicature conversationnelle violable, mais est préférée peut-être à cause de la persistance partielle de la sémantique de la construction d'origine, quelque $N$ que ce soit.

Cette étude préconise ainsi la nécessité de tenir compte, pour expliquer les propriétées distributionnelles d'un indéfini épistémique, des aspects diachroniques et des propriétés sémantiques de ses composantes, au lieu de formuler trop rapidement une explication unificatrice.

\section{Références bibliographiques}

Aloni, M. \& A. Port (2006). Epistemic Indefinites Cross-Linguistically. In Elfner, R. \& M. Walkow (eds), Proceedings of NELS, 36, 1-14.

Alonso-Ovalle, L. \& P. Menéndez Benito (2010). Modal Indefinites. Natural Language Semantics, 18.1, 1-31.

Corblin, F. (2010a). Une analyse compositionnelle de Quoi que ce soit comme universel. In Corblin, F., Tovena, L. \& E. Vlachou (éds), Langue française, 166, 17-50.

Corblin, F. (2010b). La préférence existentielle du déterminant quelque. In Tovena, L. (éd.). Déterminants en diachronie et synchronie, Paris: Projet ELICO Publications, 71-85. http://elico.linguist.univ-parisdiderot.fr/5Corblin.pdf

Culioli, A. (1982-1999). À propos de quelque. In Culioli, A. (1999). Pour une linguistique de l'énonciation. Tome 3, Paris : Ophrys, 49-58.

Doi, K. (2012). Nihongo-no <dare-ka+meesi-ku> to furansugo-no $<q u e l q u e+k a s a n-t a n s u u-m e e s i>-n o$ taisyoogenggaku-teki kenkyuu [Une étude constrastive entre la suite $<$ dare-ka+SN $>$ du japonais et $<$ quelque + nom comptable singulier $>$ du français]. Mémoire de licence. Faculté des Lettres, Université d'Okayama.

Foulet, L. (1982). Petite syntaxe de l'ancien français. Paris : Champion.

Eguchi, T. (1998). Nihongo-no kansetugimon-bun-no bonpoo-teki itizuke-nituite [On the Grammatical Status of Japanese Indirect Questions]. Kyushu University Annual Report of Linguistics, 19, 5-24.

Fălăuş, A. (2010). Alternatives as sources of semantic dependency. In Li, N. \& D. Lutz (eds), Proceedings of SALT, 20, 406-427.

Jayez, J. \& L. Tovena (2008). Evidentiality and determination. In Gronn, A. (ed.), Proceedings of Sinn und Bedeutung, 12, Oslo : University of Oslo, 271-286.

Jayez, J. \& L. Tovena (2010). Quatre problèmes pour le choix libre. In Corblin, F., Tovena, L. \& E. Vlachou (éds), Langue française, 166, 51-72.

Kadmon, N. \& F. Landman (1993). Any. Linguistics \& Philosophy, 16, 353-422.

Kamio, A. (1973). Observations on Japanese Quantifiers. Descriptive and Applied Linguistics, 6, 69-92.

Kaneko, M. (2007). Pronoms indefinis et grammaticalisation, Modèles Linguistiques, XXIII-2 : 37-57.

Kawaguchi, J. (1982). dare-ka + NP. Geibun-Kenkyuu, 44, Tokyo : Keio University, 110-126.

Kinuhata, T. \& M. Iwata (2010). Meesiku-no ka no rekisi : sengen, futee yoohoo o tyuusinni [The history of $k a$ in NP-positions: Focusing on disjunctive and indeterminate use]. Studies in Japanese Language, 6-4, 1-15.

Kinuhata, T. \& J. Whitman (2010). Genesis of Indefinite Pronouns in Japanese and Korean. In McClure, W. \& M. Den Dikken (eds), Japanese / Korean Linguistics, 18, Stanford : CSLI, 88-100.

Kratzer, A. (2005). Indefinites and the Operators they depend on: From Japanese to Salish. In Carlson, G. \& F.J. Pelletier (eds). Reference And Quantification : The Partee Effect, Stanford : CSLI, 113-142.

Kratzer, A. \& J. Shimoyama (2002). Indeterminate Pronouns: the Views from Japanese. In Otsu, Y. (ed.), The Proceedings of the Third Tokyo Conference on Psycholinguistics, Tokyo : Hituzi Pub, 1-25. 
Lefeuvre, F. (2006). Quoi de neuf sur quoi : étude morphosyntaxique du mot quoi. Rennes : Presse Universitaire de Rennes.

Muller, C. (2007). QUELQUE, déterminant singulier. Cahier de lexicologie, 90, 135-149.

Okutsu, K. (1996). Syuui Nihon-Bunpoo-Ron. Tokyo: Hituzi Pub.

Schwartzchild, R. (2002). Singleton Indefinites. Journal of Semantics, 19, 289-314.

Takahashi, D. (1994). Sluicing in Japanese. Journal of East Asian Linguistics, 3, 575-615.

Van de Velde, D. (2000). Les indéfinis comme adjectifs. In Bosveld-de Smet, L. et al. (éds). De l'indétermination à la quantification, Arras : Artois Presse Université.

Yamamori, Y. (2006). Nihongo-no genryoo-hyoogen-no kenkyuu. Tokyo : Kazama Shobo.

\begin{abstract}
${ }^{1}$ Je remercie Catherine Schnedecker de sa relecture attentive et de ses remarques précieuses. Je suis également reconnaissant à mon collègue d'Okayama, Michel de Boissieu, pour la correction du français. Toutes les insuffisances qui restent sont dues à l'auteur. Cette étude a reçu le soutien financier de la Japan Society for the
\end{abstract} Promotion of Sciences (numéro 23520463).

${ }^{2}$ Dans ce qui suit, les expressions comparables à un indéfini épistémique seront mises en gras dans chaque exemple.

3 Les abréviations utilisées sont les suivantes : ACC $=$ accusatif COMP $=$ complémenteur ; COP $=$ copule ; DAT $=$ datif ; $\mathrm{GEN}=$ génitif $; \mathrm{LOC}=$ locatif $; \mathrm{NF}=$ nom fonctionnel no $; \mathrm{NEG}=$ négation $; \mathrm{NOM}=$ nominatif $; \mathrm{PROG}=$ progressif $; \mathrm{PST}=$ passé ; $\mathrm{Q}=$ marque de question $k a ; \mathrm{SN}=$ syntagme nominal ; SUBJ $=$ subjonctif $; \mathrm{TOP}=$ topique.

${ }^{4}$ Sémantiquement, les pronoms indéterminés servent à introduire une variable, mais ne disposent pas d'une force quantificationnelle propre : dare et nani fournissent respectivement une variable d'entité humaine et une variable d'entité non-humaine ou d'événement. Dans cet article, je glose dare et nani par 'qui' et 'quoi'.

${ }^{5}$ Kaneko (2007) examine la sémantique de cette combinaison du point de vue de la grammaticalisation.

${ }^{6}$ "Quite generally, indefinite determiners might be domain shifters, operations on quantificational domains. » (Kratzer, $2005: 134$ )

${ }^{7}$ Cette présentation de la dérivation de l'inférence de libre choix est due à Fălăuş (2010).

${ }^{8}$ Schwarzchild (2002) affirme qu'un indéfini ayant le domaine singleton (qui ne contient qu'une seule alternative) donne lieu à la portée large par rapport aux autres opérateurs.

${ }^{9}$ « We would like to think of ignorance effects in EIs [=Epistemic Indefinites] as 'fossilized implicatures': inferences, pragmatic in origin, that are now part of a lexically encoded meaning. » (Aloni \& Port, $2010: 6$ ).

${ }^{10}$ Lefeuvre (2006) discute dans ce passage les indéfinis complexes, comme je ne sais quoi, on ne sait quoi, etc.

${ }^{11}$ Le jugement d'acceptabilité des contreparties françaises de (22) et (23) était très varié parmi les locuteurs natifs à qui je les ai soumises.

12 http://kuroshio.blog.ocn.ne.jp/001/2011/06/post 7854.html

${ }^{13}$ Les locuteurs qui acceptent dare-ka prénominal en (23) acceptent également la question partielle en termes de dare en (33).

${ }^{14}$ Il est toutefois à noter que Doi (2012) montre, en se basant sur les données trouvées sur Internet, que les occurrences de la lecture de libre choix sont, en japonais contemporain, deux fois plus nombreuses que celles de la lecture d'ignorance. L'auteur impute cette différence au fait que la lecture de libre choix est observée dans des environnements plus variés (non seulement avec la modalité déontique, mais aussi dans une question, un ordre, une proposition, dans l'antécédent du conditionnel, etc.), tandis que la lecture d'ignorance n'apparaît que dans des environnements limités (dans une phrase épisodique ou avec la modalité épistémique). Si cette observation est correcte, on pourrait supposer que l'interprétation originaire du dare-ka prénominal comme une question indirecte parenthétique deviendra moins dominante et que la réanalyse de cette suite comme un pronom (équivalent à la disjonction d'alternatives) s'avancera davantage. 